\title{
Does age affect symptom recovery after sports-related concussion? A study of high school and college athletes
}

\author{
Clinical article
}

\author{
*Young M. Lee, B.S.P.H., ${ }^{1}$ Mitchell J. Odom, B.S., ${ }^{1}$ Scott L. Zuckerman, M.D., ${ }^{2,3}$ \\ Gary S. Solomon, Ph.D., ${ }^{2,3}$ And Allen K. Sills, M.D. ${ }^{2,3}$ \\ ${ }^{1}$ Vanderbilt University School of Medicine, ${ }^{2}$ Department of Neurological Surgery, and ${ }^{3}$ Vanderbilt Sports \\ Concussion Center, Vanderbilt University School of Medicine, Nashville, Tennessee
}

\begin{abstract}
Object. Sport-related concussions (SRCs) in high school and college athletes represent a significant public health concern. Research suggests that younger athletes fare worse symptomatically than older athletes after an SRC. Using reliable change index (RCI) methodology, the authors conducted a study to determine if there are age-related differences in number, severity, and resolution of postconcussion symptoms.

Methods. Between 2009 and 2011, baseline measures of neurocognitive functions and symptoms in high school and college athletes were entered into a regional database. Seven hundred forty of these athletes later sustained an SRC. Ninety-two athletes in the 13- to 16-year-old group and 92 athletes in the 18- to 22-year-old group were matched for number of prior concussions, sex, biopsychosocial variables, and days to first postconcussion testing and symptom assessment. A nonparametric Mann-Whitney U-test was used to compare the severity of each of 22 symptoms comprising the Total Symptom Scale (TSS) at baseline and first postconcussion test. To obtain a familywise $p$ value of 0.05 for each test, the significance level for each symptom comparison was set at an alpha of $0.05 / 22$ $=0.0023$. The number of days to return to baseline TSS score was compared using the RCI methodology, set at the $80 \%$ confidence interval, equal to a change in raw score of 9.18 points on the TSS.

Results. There was no statistically significant difference in symptom presence, symptom severity, and total symptoms between the age groups at baseline or at postconcussion testing. There was no statistically significant difference in return to baseline symptom scores between the age groups.

Conclusions. Using RCI methodology, there was no statistically significant difference between younger and older athletes in return to baseline symptoms postconcussion.

(http://thejns.org/doi/abs/10.3171/2013.7.PEDS12572)
\end{abstract}

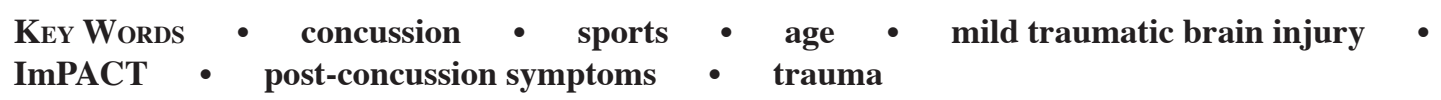

$\mathrm{C}$ oncussion, also termed "mild traumatic brain injury," is a complex pathophysiological process induced by traumatic biomechanical forces to the brain. ${ }^{3}$ In the US, 1.6-3.2 million traumatic brain injuries (TBIs) occur each year, with the majority classified as mild and over $50 \%$ occurring in children. ${ }^{11}$ More than 300,000 TBIs result from sports-related activities each year in the US. ${ }^{33}$ Sports-related concussion (SRC) has been found to account for more than $10 \%$ of all athleticsrelated injuries in high school athletes. ${ }^{19,25}$ From 2001 to 2009, emergency department visits for SRC in children

\footnotetext{
Abbreviations used in this paper: CIS = Concussion in Sport; ImPACT $=$ Immediate Post-Concussion Assessment and Cognitive Testing; RCI = reliable change index; SRC $=$ sport-related concussion; TBI = traumatic brain injury; TSS = Total Symptom Scale.

* Mr. Lee and Mr. Odom contributed equally to this work.
}

and adolescents increased by over $60 \%$, with the highest rate of SRC emergency department visits occurring in individuals aged $10-14$ years. ${ }^{20}$ Sports-related concussion represents a growing problem that affects athletes at the middle school, high school, collegiate, and professional levels.

Data from the National Athletic Trainers' Association from 1995 to 1997 indicate a high school SRC rate of $3.6 \%$ compared with a collegiate SRC rate of $4.8 \% . .^{14,28}$ Most recently, Gessel et al. reported that the incidence of SRC among high school athletes was 3\%-6\%.18 Concussions make up nearly twice the proportion of total injuries at the high school level compared with the collegiate level. ${ }^{18}$ These differences in SRC rates at the high school and collegiate levels indicate a possible disparity in injury patterns between different age groups.

At the 3rd Concussion in Sport (CIS) group meeting 
held in Zurich in $2008,{ }^{27}$ international concussion experts agreed that an athlete's age represented a "modifying" factor having the potential to influence concussion management and predict a protracted recovery. The consensus was that age less than 18 years is a modifying factor. Although there was unanimous agreement that the evaluation and management recommendations could be applied to children and adolescents as young as 10 years old, the CIS group concluded that "because of the different physiological response and longer recovery after concussion and specific risks related to head impact during childhood and adolescence, a more conservative return to play approach is recommended." ${ }^{27}$ Middle and high school athletes are at an earlier stage of neural development, with concussion possibly leading to delayed recovery. ${ }^{16}$ High school and youth athletes are of special concern to those engaged in the SRC debate.

Symptom management is a cornerstone of concussion treatment. Commonly reported postconcussion symptoms include, but are not limited to, headaches, dizziness, fatigue, irritability, reduced concentration, sleep disturbance, memory dysfunction, anxiety, sensitivity to noise, double or blurred vision, sensitivity to light, and depression. ${ }^{1,5,29}$ Symptom resolution is often the final factor taken into account when returning an athlete to play. ${ }^{6}$ Although most of these symptoms resolve in a short period of time after the injury, a minority of individuals suffer from long-term sequelae even 12 months after injury. ${ }^{1,9,15}$ Postconcussion syndrome in the young athlete can result in his or her removal from play and lead to reactive depression and interference with school and extracurricular activities. ${ }^{8}$ Young athletes can have wide variation in physiological and neurobehavioral symptoms after SRC..$^{23}$

Controversy exists regarding the relationship between age and symptoms of concussion. Covassin et al..$^{13}$ tested 837 college and 779 high school athletes and found that high school athletes reported more baseline somatic/migraine symptoms than college athletes, whereas college athletes reported more emotional and sleep symptoms than high school athletes. ${ }^{13}$ All participants with a history of TBI were excluded, and return to baseline symptoms after SRC was not investigated. In a separate, prospective study investigating the effect of age on symptom recovery after SRC, Covassin and colleagues ${ }^{12}$ evaluated 150 high school and 72 college athletes who sustained concussions. The authors found no difference in total symptoms by age group, and they reported that both high school and college athletes returned to baseline symptom scores by 14 days. ${ }^{12}$ This study did not control for the participants' concussion history.

However, a retrospective observational study by Cantu et al. ${ }^{7}$ did find an association between age and duration of symptoms in 145 concussed athletes younger than 18 years of age and 70 concussed athletes 18 years or older. The older cohort had a greater average duration of symptoms, although there was no age-related difference surrounding the presence of symptoms and number of symptoms. A history of concussion was not used as an exclusion criterion. Conflicting results have been reported by Field et al. ${ }^{16}$ who found that high school athletes with a concussion had a significant increase in postconcussion symptoms relative to control subjects on Days 1,3 , and 5 after injury, while college students had no significant differences by Day 5. These results seem inconsistent given that a history of multiple concussions has also been linked to more concussion-related symptoms at the time of baseline testing ${ }^{30}$ and that college athletes had a significantly higher rate of concussions in this study. ${ }^{16} \mathrm{~A}$ synthesis of the aforementioned studies suggests that prior concussion may be a relevant factor in the endorsement of postconcussion symptoms at both the high school and collegiate levels.

We endeavored to assess for age differences in postconcussion symptoms and time to return to symptom baseline following SRC in matched cohorts of 13- to 16-year-old and 18- to 22-year-old athletes. Despite the fact that Covassin et al. ${ }^{13}$ found no differences between age groups and a number of conflicting results, we based our hypothesis on the most recent CIS consensus statement that regarded age as a predictor of a protracted recovery. We hypothesized that younger athletes, compared with older athletes, would: 1) endorse more symptoms postconcussion, 2) endorse increased symptom severity postconcussion, and 3) experience increased average time to return to symptom baseline as measured by the Total Symptom Scale (TSS).

\section{Methods}

\section{Study Design}

Institutional review board approval was obtained prior to data collection. Our study has a retrospective, observational design. Participants were recruited from high schools and colleges in western Pennsylvania between 2009 and 2011 as part of a regional neurocognitive testing program. Some participants were recruited from middle schools; these student athletes are included in the high school group. Data were collected primarily by certified athletic trainers and doctoral level neuropsychologists at high schools and colleges and in a sports concussion specialty clinic in the Pittsburgh metropolitan area.

After written informed consent was obtained from the athlete or his/her parent/guardian, all athletes completed a baseline symptom inventory questionnaire, the TSS, which is included in the ImPACT (Immediate PostConcussion Assessment and Cognitive Testing) software $^{26}$ as part of routine athletic care. The TSS was completed prior to sport participation at each athlete's school, in a controlled group-setting environment with minimal distractions. All ImPACT tests were proctored and administered in an isolated, quiet room, in a group setting.

\section{Data Collection}

We chose to use the ImPACT battery to obtain baseline symptom data. All patients took postconcussion ImPACT tests as part of routine clinical care and were matched with other participants on the number of days to first postconcussion test. ImPACT is a commercially available computerized test for SRC that provides symptom and neurocognitive test data. ${ }^{26}$ In addition to providing neurocognitive scores, ImPACT's TSS component is a concussion symptom inventory consisting of 22 items, 
each rated on a 7-point Likert scale (Scores 0-6), that assess for the presence and severity of symptoms after a concussion. ${ }^{24}$ The TSS scores can be reported in terms of number of symptoms present (range 0-22) or in terms of symptom severity (range 0-132). Previous studies have shown the TSS to be valid ${ }^{10,31}$ and reliable relative to SRC assessment. ${ }^{24}$ In the current study, raw scores were recorded for the TSS.

As pertains to our hypothesis, 3 key distinctions were made when evaluating an athlete's symptomatology:

1) Symptom presence: this was an all or none, dichotomous phenomenon for each individual symptom. Athletes either had a symptom, with a score of 1-6, or did not have a symptom, with a score of 0 .

2) Severity of symptoms: this was a graded phenomenon for each individual symptom. Athletes endorsed the severity of each symptom, with 6 possible gradations of that specific symptom, from 1 to 6 .

3) Total symptoms: the gradation of individual symptoms was not of concern. Rather, we focused on the total number of symptoms. If Athlete A endorsed only headache and nausea, while Athlete B endorsed only photophobia and inattention, the total symptom score in both patients would be the same regardless of their individual symptoms.

The timing of the administration of ImPACT testing was dictated by clinical factors rather than by a standardized research protocol. The primary dependent variable was operationally defined as the number of days until the TSS score returned to the participant's own baseline for the TSS indexes. Using reliable change index (RCI) methodology set at the $80 \%$ confidence interval, ${ }^{21}$ raw change scores equal to or greater than 9.18 points for TSS symptom severity met criteria for a statistically significant change. Any difference between postconcussion symptom severity score and baseline less than this value was defined as a return to baseline.

\section{Selection of Participants}

Following head trauma, the diagnosis of concussion was established based on the following on-field or sideline signs or symptoms: 1) lethargy, fogginess, headache, and such; 2) alteration in mental status; 3) loss of consciousness; or 4) amnesia. The diagnosis of concussion was made by an athletic trainer or team physician. Following the recommendations of the CIS consensus guidelines, no grading system was used to rate the severity of the concussion.

The inclusion criteria for the current study were as follows: 1) age 13-16 years or 18-22 years at time of concussion, 2) enrollment as a high school (in some cases middle school) or college athlete, 3 ) valid completion of up to 2 postconcussion ImPACT testing data points, and 4) fluency in English. Exclusion criteria were as follows: 1) ages less than 13, equal to 17 , or greater than 22 years; 2) self-reported history of special education, speech therapy, repeated year(s) of school, learning disability, attention deficit hyperactivity disorder, dyslexia, or autism; 3) self-reported history of brain surgery or seizure disorder; and 4) self-reported history of treatment for drug/alcohol abuse or psychiatric illness. We purposely excluded athletes 17 years of age to clearly delineate the 2 cohorts based on age.

\section{Matching of Age Cohorts}

The final sample of the study participants was formulated by the following process. From the regional database of athletes, we identified 740 participants who had previously completed valid baseline ImPACT testing and then gone on to suffer an SRC. An invalid baseline test was defined operationally as an ImPACT Impulse Control Composite score of greater than 30 . Of these 740 athletes, 126 were outside of the age range of our study, and 112 had a history of special education, speech therapy, repeated year of school, or learning disability and were excluded from the study. Of the remaining 502 eligible participants who sustained a concussion during the study period, 184 (92 in the 13- to 16-year range and 92 in the 18- to 22-year range) who had completed at least 2 postconcussion ImPACT tests were matched based on the number of prior concussions and were subsequently included in the study. A flow chart showing the breakdown of patients included in the study is shown in Fig. 1. The 13- to 16-year-old and 18- to 22-year-old cohorts all met inclusion criteria and were successfully matched based on the number of prior concussions.

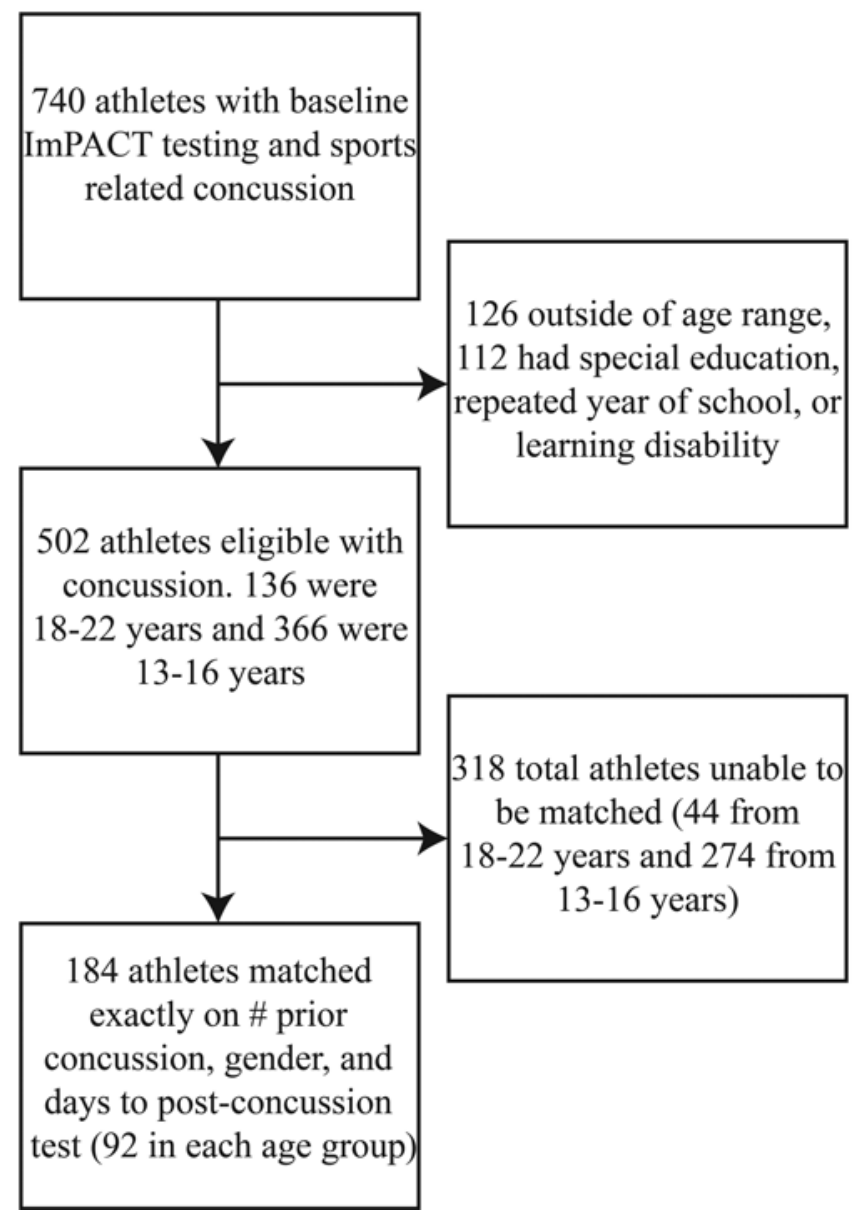

Fig. 1. Flow chart showing participant screening and matching for the age-comparison study arm. 


\section{Statistical Analysis}

Descriptive statistics are reported as mean \pm SD for continuous variables and as frequency and proportion for categorical variables. The proportion of participants endorsing symptom presence was compared between the 2 age groups using Fisher exact and chi-square tests. Viewed as means and standard deviations, the severity of individual symptoms and the total symptom score (calculated as the sum of severities of individual symptom scores) for both age groups were assessed at baseline and at first postconcussion test date and compared using the Mann-Whitney U-test. The significance of the difference between the 2 age groups for the aforementioned measures was evaluated according to a Bonferroni correction at an alpha of $0.05 / 22=0.0023$. For participants whose ImPACT TSS score returned to baseline within the 30-day study period, we calculated the number of days it took the score to return to baseline, using the RCI at the $80 \%$ confidence level. For each symptom score, the mean number of days it took for the score to return to baseline was compared between the 2 age groups using a Mann-Whitney U-test, and the significance of this difference was evaluated at $\alpha=0.05$. None of the cases included in the final analyses had any missing data. Statistical analyses were performed using IBM SPSS Statistics, version 20.0.0 (IBM Corp., 2011). Mean results are presented $\pm \mathrm{SD}$.

\section{Results}

By design, there was a difference in the average age and number of years of education between the age groups. The younger group averaged $15.0 \pm 0.8$ years of age and $8.6 \pm 1.0$ years of education, and the older group averaged $19.1 \pm 1.1$ years of age and $12.1 \pm 1.5$ years of education. Furthermore, as might be expected given the group age differences, there were differences in physical characteristics. The mean height, weight, and body mass index were $66.2 \pm 3.4$ inches, $139.8 \pm 30.6$ pounds, and $22.3 \pm 3.6$ for the younger age group, respectively, and $68.6 \pm 4.3$ inches, $166.6 \pm 42.6$ pounds, and $24.7 \pm 4.2$ for the older age group, respectively. The groups were matched for sex, and thus there was no difference in sex composition, with $52 \mathrm{fe}$ males $(56.5 \%)$ in each group. Matching also was completed with concussion history, and both groups had an average of $0.2 \pm 0.6$ prior concussions. The distribution of the type of sport resulting in the concussion did not differ between these groups. These results are summarized in Table 1.

TABLE 1: Demographic characteristics of 184 participants

\begin{tabular}{|c|c|c|c|}
\hline \multirow[b]{2}{*}{ Characteristic } & \multicolumn{2}{|c|}{ Age Group } & \multirow[b]{2}{*}{$\mathrm{p}$ Value } \\
\hline & $13-16$ Yrs $(n=92)$ & $18-22$ Yrs $(n=92)$ & \\
\hline mean age* & $15.0 \pm 0.8$ & $19.1 \pm 1.1$ & $<0.001$ \\
\hline female sex $\dagger$ & $52(56.5)$ & $52(56.5)$ & $>0.999$ \\
\hline \multicolumn{4}{|l|}{ handedness $†$} \\
\hline right-handedness & $87(94.6)$ & $78(84.8)$ & \\
\hline ambidexterity & $2(2.2)$ & $4(4.3)$ & 0.085 \\
\hline mean height (inches) ${ }^{*}$ & $66.2 \pm 3.4$ & $68.6 \pm 4.3$ & $<0.001$ \\
\hline mean weight $(\mathrm{lbs})^{*}$ & $139.8 \pm 30.6$ & $166.6 \pm 42.6$ & $<0.001$ \\
\hline mean body mass index ${ }^{*}$ & $22.3 \pm 3.6$ & $24.7 \pm 4.2$ & $<0.001$ \\
\hline mean no. of yrs of education* & $8.6 \pm 1.0$ & $12.1 \pm 1.5$ & $<0.001$ \\
\hline mean no. of prior concussions ${ }^{*}$ & $0.2 \pm 0.6$ & $0.2 \pm 0.6$ & $>0.999$ \\
\hline \multicolumn{4}{|l|}{ type of sport $\dagger$} \\
\hline soccer & $24(26.1)$ & $23(25.0)$ & \\
\hline football & $26(28.3)$ & $25(27.2)$ & \\
\hline basketball & $8(8.7)$ & $16(17.4)$ & \\
\hline tennis & $0(0.0)$ & $2(2.2)$ & \\
\hline ice hockey & $3(3.3)$ & $2(2.2)$ & \\
\hline volleyball & $10(10.9)$ & $2(2.2)$ & \\
\hline baseball & $1(1.1)$ & $2(2.2)$ & \\
\hline softball & $3(3.3)$ & $7(7.6)$ & \\
\hline cheerleading & $2(2.2)$ & $4(4.3)$ & \\
\hline lacrosse & $7(7.6)$ & $7(7.6)$ & \\
\hline field hockey & $4(4.3)$ & $2(2.2)$ & \\
\hline swimming & $1(1.1)$ & $0(0.0)$ & \\
\hline mountain biking & $1(1.1)$ & $0(0.0)$ & \\
\hline unknown & $2(2.2)$ & $0(0.0)$ & 0.177 \\
\hline
\end{tabular}


Age-related and sports-related postconcussion symptoms

\section{Symptom Presence: Baseline and Postconcussion}

At baseline, there were no statistically significant group differences in the number of symptoms (a self-reported symptom score $>0$ ) for headache, nausea, vomiting, balance problems, dizziness, fatigue, trouble falling asleep, sleeping more than usual, sleeping less than usual, drowsiness, sensitivity to light, sensitivity to noise, irritability, sadness, nervousness, feeling more emotional, numbness or tingling, feeling slowed down, feeling mentally foggy, difficulty concentrating, difficulty remembering, and visual problems.

There were also no statistically significant intergroup differences in the number of postconcussion symptoms. Both younger and older age groups reported a similar total number of symptoms at both baseline and at the postconcussion time points. These results are presented in Tables 2 and 3.

\section{Symptom Severity: Baseline and Postconcussion}

There were no age-based differences in the severity of baseline symptoms as determined by assessing the average of the self-reported symptom score (from 0 to 6).

TABLE 2: Presence of symptoms at baseline testing

\begin{tabular}{lccr}
\hline \multicolumn{1}{c}{ Symptom } & \multicolumn{2}{c}{ Age Group* } & \\
\cline { 2 - 3 } \multicolumn{1}{c}{ Younger } & Older & \\
\cline { 2 - 3 } & $(\mathrm{n}=92)$ & $(\mathrm{n}=92)$ & $\mathrm{p}$ Value† \\
\hline headache & $24(26.1)$ & $18(19.6)$ & 0.380 \\
nausea & $4(4.3)$ & $3(3.3)$ & $>0.999$ \\
vomiting & $2(2.2)$ & $1(1.1)$ & $>0.999$ \\
balance problems & $7(7.6)$ & $3(3.3)$ & 0.330 \\
dizziness & $14(15.2)$ & $5(5.4)$ & 0.050 \\
fatigue & $19(20.7)$ & $22(23.9)$ & 0.723 \\
trouble falling asleep & $22(23.9)$ & $19(20.7)$ & 0.723 \\
sleeping more than usual & $7(7.6)$ & $9(9.8)$ & 0.795 \\
sleeping less than usual & $26(28.3)$ & $16(17.4)$ & 0.113 \\
drowsiness & $25(27.2)$ & $18(19.6)$ & 0.296 \\
sensitivity to light & $11(12.0)$ & $5(5.4)$ & 0.190 \\
sensitivity to noise & $5(5.4)$ & $5(5.4)$ & $>0.999$ \\
irritability & $20(21.7)$ & $15(16.3)$ & 0.453 \\
sadness & $13(14.1)$ & $11(12.0)$ & 0.827 \\
nervousness & $25(27.2)$ & $17(18.5)$ & 0.219 \\
feeling more emotional & $15(16.3)$ & $19(20.7)$ & 0.569 \\
numbness or tingling & $9(9.8)$ & $5(5.4)$ & 0.405 \\
feeling slowed down & $11(12.0)$ & $6(6.5)$ & 0.309 \\
feeling mentally foggy & $14(15.2)$ & $5(5.4)$ & 0.050 \\
difficulty concentrating & $21(22.8)$ & $16(17.4)$ & 0.462 \\
difficulty remembering & $10(10.9)$ & $10(10.9)$ & $>0.999$ \\
visual problems & $10(10.9)$ & $3(3.3)$ & 0.081 \\
average no. of symptoms $\neq$ & $3.4(3.9)$ & $2.5(3.7)$ & 0.111 \\
\hline
\end{tabular}

* Values are presented as the number (\%) of patients with a given symptom. Some patients had more than one symptom.

$\dagger$ A family-wise $p$ value of 0.05 was obtained using a Bonferroni corrected significance level of $\alpha=0.05 / 22=0.0023$.

$\ddagger$ Average number of symptoms per patient.
TABLE 3: Presence of symptoms at postconcussion testing

\begin{tabular}{lccc}
\hline \multirow{2}{*}{\multicolumn{1}{c}{ Symptom }} & \multicolumn{2}{c}{ Age Group* } & \\
\cline { 2 - 3 } & Younger & Older & \\
& $(\mathrm{n}=92)$ & $(\mathrm{n}=92)$ & p Value† \\
\hline headache & $78(84.8)$ & $65(70.7)$ & 0.033 \\
nausea & $27(29.3)$ & $24(26.1)$ & 0.742 \\
vomiting & $5(5.4)$ & $0(0.0)$ & 0.059 \\
balance problems & $43(46.7)$ & $33(35.9)$ & 0.178 \\
dizziness & $50(54.3)$ & $38(41.3)$ & 0.104 \\
fatigue & $51(55.4)$ & $52(56.5)$ & $>0.999$ \\
trouble falling asleep & $35(38.0)$ & $28(30.4)$ & 0.351 \\
sleeping more than usual & $23(25.0)$ & $19(20.7)$ & 0.599 \\
sleeping less than usual & $25(27.2)$ & $16(17.4)$ & 0.156 \\
drowsiness & $55(59.8)$ & $46(50.0)$ & 0.236 \\
sensitivity to light & $48(52.2)$ & $40(43.5)$ & 0.302 \\
sensitivity to noise & $45(48.9)$ & $33(35.9)$ & 0.101 \\
irritability & $31(33.7)$ & $23(25.0)$ & 0.257 \\
sadness & $17(18.5)$ & $14(15.2)$ & 0.694 \\
nervousness & $17(18.5)$ & $15(16.3)$ & 0.846 \\
feeling more emotional & $18(19.6)$ & $24(26.1)$ & 0.380 \\
numbness or tingling & $7(7.6)$ & $4(4.3)$ & 0.536 \\
feeling slowed down & $40(43.5)$ & $32(34.8)$ & 0.290 \\
feeling mentally foggy & $45(48.9)$ & $36(39.1)$ & 0.235 \\
difficulty concentrating & $52(56.5)$ & $54(58.7)$ & 0.881 \\
difficulty remembering & $31(33.7)$ & $24(26.1)$ & 0.334 \\
visual problems & $23(25.0)$ & $21(22.8)$ & 0.863 \\
average no. of symptoms & $8.3(5.9)$ & $7.0(5.3)$ & 0.101 \\
\hline
\end{tabular}

* Values are presented as the number (\%) of patients with a given symptom.

† A family-wise $p$ value of 0.05 was obtained using a Bonferroni corrected significance level of $\alpha=0.05 / 22=0.0023$.

$\ddagger$ Average number of symptoms per patient.

No between-group differences were found for postconcussion symptom severity scores. There were also no intergroup differences in total symptom score at baseline or after concussion in terms of total symptom score. These results are presented in Tables 4 and 5 .

\section{Return to Baseline Score of Total Symptoms After Concussion}

Symptom scores in $88(95.7 \%)$ of the younger participants and $89(96.7 \%)$ of the older participants returned to baseline within 30 days of concussion ( $p>0.999)$.

For patients in whom scores returned to baseline postconcussion, the time to return to baseline was similar between both age groups, with the younger group averaging $6.9 \pm 5.5$ days and the older group averaging $5.7 \pm 4.1$ days $(\mathrm{p}=0.087)$. Although this did not reach our threshold of 0.05 , it bordered on statistical significance. These results are presented in Table 6.

\section{Discussion}

The Concussion in Sport group has indicated that 
Y. M. Lee et al.

TABLE 4: Severity of symptoms at baseline testing

\begin{tabular}{|c|c|c|c|}
\hline \multirow[b]{2}{*}{ Symptom } & \multicolumn{2}{|c|}{ Age Group* } & \multirow[b]{2}{*}{$\mathrm{p}$ Value } \\
\hline & $\begin{array}{l}\text { Younger } \\
(n=92)\end{array}$ & $\begin{array}{l}\text { Older } \\
(n=92)\end{array}$ & \\
\hline headache & $0.55(1.10)$ & $0.41(1.01)$ & 0.365 \\
\hline nausea & $0.09(0.48)$ & $0.05(0.34)$ & 0.599 \\
\hline vomiting & $0.02(0.15)$ & $0.02(0.21)$ & $>0.999$ \\
\hline balance problems & $0.10(0.37)$ & $0.03(0.18)$ & 0.126 \\
\hline dizziness & $0.26(0.82)$ & $0.07(0.29)$ & 0.034 \\
\hline fatigue & $0.37(0.85)$ & $0.51(1.05)$ & 0.318 \\
\hline trouble falling asleep & $0.61(1.28)$ & $0.51(1.14)$ & 0.584 \\
\hline sleeping more than usual & $0.29(1.17)$ & $0.21(0.72)$ & 0.545 \\
\hline sleeping less than usual & $0.74(1.38)$ & $0.47(1.24)$ & 0.161 \\
\hline drowsiness & $0.51(1.03)$ & $0.43(1.05)$ & 0.621 \\
\hline sensitivity to light & $0.29(0.97)$ & $0.14(0.60)$ & 0.202 \\
\hline sensitivity to noise & $0.12(0.57)$ & $0.12(0.53)$ & $>0.999$ \\
\hline irritability & $0.37(0.79)$ & $0.32(0.81)$ & 0.647 \\
\hline sadness & $0.16(0.43)$ & $0.27(0.92)$ & 0.304 \\
\hline nervousness & $0.45(0.87)$ & $0.43(1.14)$ & 0.942 \\
\hline feeling more emotional & $0.33(0.92)$ & $0.48(1.10)$ & 0.310 \\
\hline numbness or tingling & $0.15(0.61)$ & $0.05(0.23)$ & 0.152 \\
\hline feeling slowed down & $0.23(0.79)$ & $0.09(0.35)$ & 0.118 \\
\hline feeling mentally foggy & $0.16(0.40)$ & $0.10(0.45)$ & 0.298 \\
\hline difficulty concentrating & $0.54(1.24)$ & $0.45(1.14)$ & 0.578 \\
\hline difficulty remembering & $0.18(0.61)$ & $0.22(0.72)$ & 0.741 \\
\hline visual problems & $0.24(0.76)$ & $0.05(0.34)$ & 0.036 \\
\hline mean symptom scoreł & $6.77(9.02)$ & $5.43(9.64)$ & 0.333 \\
\hline
\end{tabular}

* Values represent mean symptom severity scores (SD) on a scale from 1 to 6.

$\dagger$ A family-wise $p$ value of 0.05 was obtained using a Bonferroni corrected significance level of $\alpha=0.05 / 22=0.0023$.

$\ddagger$ Mean symptom severity score per patient.

younger age is a modifying factor in SRC. ${ }^{27}$ Prior empirical studies of age-related symptoms reported post-SRC have yielded mixed results., ${ }^{72,13,16}$ These prior studies have not systematically matched groups on the demographic variables of concussion history, sex, sport, and time of testing postinjury. We endeavored to assess acute symptom differences after SRC in 2 different age groups, taking into account the additional demographic variables described in Methods. We hypothesized that, compared with older athletes, younger athletes would 1) endorse more symptoms postconcussion, 2) endorse increased symptom severity postconcussion, and 3) have an increased average time to return to symptom baseline, as measured by the TSS. We found the following: First, there were no intergroup differences of significance at baseline. Second, younger and older athletes experienced an equal number of symptoms after SRC. Third, younger and older athletes experienced equal symptom severity after SRC. Fourth, younger athletes took an average of 1.3 days longer to return to their preconcussion total symptom profile, which bordered on (but did not meet) the conventional level of statistical significance $(\mathrm{p}=0.087)$. These results
TABLE 5: Severity of symptoms at postconcussion testing

\begin{tabular}{lccc}
\hline \multirow{2}{*}{\multicolumn{1}{c}{ Symptom }} & \multicolumn{2}{c}{ Age Group* } & \\
\cline { 2 - 3 } \multicolumn{1}{c}{ Younger } & Older & \\
\cline { 2 - 3 } headache & $(\mathrm{n}=92)$ & $(\mathrm{n}=92)$ & p Value† \\
nausea & $2.51(1.59)$ & $2.20(1.77)$ & 0.206 \\
vomiting & $0.74(1.36)$ & $0.62(1.31)$ & 0.544 \\
balance problems & $0.07(0.29)$ & $0.00(0.00)$ & 0.033 \\
dizziness & $0.96(1.28)$ & $0.74(1.20)$ & 0.236 \\
fatigue & $1.15(1.43)$ & $1.04(1.54)$ & 0.620 \\
trouble falling asleep & $1.62(1.85)$ & $1.58(1.69)$ & 0.868 \\
sleeping more than usual & $1.03(1.54)$ & $0.84(1.48)$ & 0.381 \\
sleeping less than usual & $0.62(1.33)$ & $0.58(1.30)$ & 0.823 \\
drowsiness & $0.73(1.41)$ & $0.57(1.39)$ & 0.431 \\
sensitivity to light & $1.41(1.54)$ & $1.47(1.75)$ & 0.823 \\
sensitivity to noise & $1.11(1.38)$ & $0.98(1.37)$ & 0.520 \\
irritability & $1.07(1.36)$ & $0.88(1.37)$ & 0.358 \\
sadness & $0.72(1.21)$ & $0.62(1.27)$ & 0.592 \\
nervousness & $0.23(0.52)$ & $0.43(1.22)$ & 0.136 \\
feeling more emotional & $0.32(0.81)$ & $0.39(1.07)$ & 0.587 \\
numbness or tingling & $0.36(0.83)$ & $0.70(1.46)$ & 0.056 \\
feeling slowed down & $0.13(0.54)$ & $0.08(0.40)$ & 0.438 \\
feeling mentally foggy & $0.93(1.34)$ & $0.73(1.25)$ & 0.281 \\
difficulty concentrating & $1.00(1.28)$ & $0.95(1.43)$ & 0.786 \\
difficulty remembering & $1.51(1.73)$ & $1.46(1.61)$ & 0.826 \\
visual problems & $0.64(1.14)$ & $0.46(0.87)$ & 0.217 \\
mean symptom scoreł & $0.55(1.14)$ & $0.43(0.91)$ & 0.432 \\
\hline & $19.40(18.25)$ & $17.72(18.18)$ & 0.531 \\
\hline
\end{tabular}

* Values represent mean symptom severity scores (SD) on a scale from 1 to 6.

$\dagger$ A family-wise $p$ value of 0.05 was obtained using a Bonferroni corrected significance level of $\alpha=0.05 / 22=0.0023$.

$\ddagger$ Mean symptom severity score per patient.

support the finding that younger and older athletes exhibit similar symptom profiles following SRC, with a slightly increased time to return to symptom baseline in younger athletes.

As mentioned in the earlier discussion of age-related symptoms, the evidence to date has been conflicting, with several studies showing age differences in post-SRC

TABLE 6: Return to baseline TSS score using RCl scores set at the $80 \%$ confidence interval

\begin{tabular}{lccr}
\hline & \multicolumn{2}{c}{ Age Group } & \\
\cline { 2 - 3 } \multicolumn{1}{c}{ Return to Baseline } & $\begin{array}{c}\text { Younger } \\
(\mathrm{n}=92)\end{array}$ & $\begin{array}{c}\text { Older } \\
(\mathrm{n}=92)\end{array}$ & p Value \\
\hline no. to baseline $\leq 30$ days* & $88(95.7)$ & $89(96.7)$ & $>0.999$ \\
mean days to 1st test $\dagger$ & $3.14 \pm 2.31$ & $3.14 \pm 2.31$ & $>0.999$ \\
mean days to 2nd test $\dagger$ & $9.93 \pm 4.84$ & $8.63 \pm 5.02$ & 0.021 \\
mean days to baseline $\dagger$ & $6.92 \pm 5.50$ & $5.66 \pm 4.10$ & 0.087 \\
\hline
\end{tabular}

* Values are number (\%) of patients.

$\dagger$ Mean values are presented \pm SD. 
symptomatology. ${ }^{7,12,13,16}$ However, we found no such differences in our study. In testing mean scores between the groups, prior studies have shown a difference in younger versus older athletes' symptom endorsement. Previous studies have not used RCI methodology. Reliable change index-based scores in SRC research use an athlete as his/ her own control, as opposed to focusing on mean group differences. These scores take into account normal variability in symptoms from time point to time point, testretest reliability of the measure employed, and other typical sources of error variance found in the measurement of human cognition and behavior. Symptoms are states and vary from day to day, with or without concussion injury. Utilizing RCI-based scores is one method of accounting for potentially extraneous sources of variance in symptom reporting. It may be useful for the sports medicine clinician to use RCI-based scores when addressing the symptom component of the SRC puzzle because reliance on number and/or ratings of symptom severity may not capture the symptom picture accurately.

As the incidence of SRC has risen over the past decade, so has awareness of and concern for athletes participating at all skill levels. Because of their level of neurological development, younger athletes may be at an inherently higher risk for acute neurocognitive deficits and more severe postconcussion symptom profiles. However, based on new results, some authors have proposed that long-term SRC outcomes should also be considered. Recently, there have been studies indicating that the effects of mild TBI and SRC in adults ${ }^{2,17}$ and professional athletes ${ }^{22}$ may extend beyond the days it takes to return to neurocognitive and symptom baseline. These reports have created much more concern for the incidence of SRC in children and children's subsequent recovery. However, we believe that the question of the long-term effects of mild TBI and SRC will be addressed best by prospective, controlled, and longitudinal studies utilizing multimodal assessment methodologies. Until the results of such studies are available, we can only advise caution in reaching premature or unfounded conclusions.

Although the focus of our study was on postconcussion symptom profile and return to symptom baseline, several authors have published work on neurocognitive testing following SRC in various age groups. These studies, relating neurocognitive deficits and return to neurocognitive baseline scores, have provided some of the only age-related data on SRC outcome and have produced inconsistent results regarding time to return to neurocognitive baseline..$^{4,16,32,34}$ Due to conflicting findings, further categorization is needed to stratify the effect of age on SRC. Our study aimed to further elucidate age disparities in concussion management by analyzing symptoms post-SRC.

The present study has several methodological strengths. First, many demographic variables previously not systematically controlled for in prior studies were addressed here. We controlled for sex, concussion history, multiple biopsychosocial variables, and time of testing postconcussion. We controlled generally, but not specifically, for the variable of sport as a between-group factor. We addressed variations of the symptom variable, includ- ing presence of a symptom, symptom severity, and total score. We found that when these variables were controlled for adequately, the previously noted age-related symptom differences reported in prior studies disappeared. A Bonferroni correction was applied to individual symptom comparisons to ensure that the Type I error rate for comparing symptom presence and severity at baseline and post-SRC testing was correctly adjusted to a significance level of 0.05 . In addition, we used RCI-based scores, as opposed to group means, which assessed the athletes' return to their own baseline TSS score and eliminated the effect of between-group and between-individual differences in baseline TSS scores.

Conversely, our study has limitations. First, this was not a prospective study but a retrospective cohort study of a convenience sample of clinical participants. Second, there was no standardized protocol for a post-SRC testing timeline. Participants included in the study completed 2 post-SRC ImPACT/TSS tests, although it was not required of them to be tested at specific intervals post-SRC. This aspect reflects the clinical nature of the study and the variable practices of sports medicine clinicians. We sought to standardize the testing interval by including the number of days between the concussion and the first post-SRC ImPACT test as a criterion used for matching. Accordingly, the number of days between concussion and return to baseline in each individual athlete was assessed directly as the outcome variable. Third, it is possible that our statistical criteria may have been too stringent. A larger sample would allow for this determination, but our rigorous matching criteria precluded the use of a larger sample. Finally, all study participants were from one region of the country and may not represent practice in other regions of the United States.

\section{Conclusions}

Using RCI methodology in a closely matched cohort of athletes, there was no statistically significant difference between younger and older athletes in symptom presence, symptom severity, and total symptoms at baseline and postconcussion testing. In addition, there was no difference between younger and older athletes in return to symptom baseline postconcussion.

\section{Disclosure}

Dr. Solomon reports being a consultant for ImPACT.

Author contributions to the study and manuscript preparation include the following. Conception and design: Zuckerman, Solomon. Acquisition of data: Zuckerman, Solomon, Sills. Analysis and interpretation of data: Zuckerman, Lee, Solomon. Drafting the article: Zuckerman, Lee, Odom. Critically revising the article: all authors. Reviewed submitted version of manuscript: all authors. Approved the final version of the manuscript on behalf of all authors: Zuckerman. Statistical analysis: Lee. Administrative/technical/material support: Solomon, Sills. Study supervision: Zuckerman, Solomon, Sills.

\section{References}

1. Alves WM, Colohan AR, O'Leary TJ, Rimel RW, Jane JA: Understanding posttraumatic symptoms after minor head injury. J Head Trauma Rehabil 1:1-12, 1986 
2. Anderson V, Brown S, Newitt H, Hoile H: Long-term outcome from childhood traumatic brain injury: intellectual ability, personality, and quality of life. Neuropsychology 25:176-184, 2011

3. Aubry M, Cantu R, Dvorak J, Graf-Baumann T, Johnston K, Kelly J, et al: Summary and agreement statement of the first international conference on concussion in sport, Vienna 2001. Phys Sportsmed 30:57-63, 2002

4. Baillargeon A, Lassonde M, Leclerc S, Ellemberg D: Neuropsychological and neurophysiological assessment of sport concussion in children, adolescents and adults. Brain Inj 26:211-220, 2012

5. Bohnen N, Twijnstra A, Jolles J: Post-traumatic and emotional symptoms in different subgroups of patients with mild head injury. Brain Inj 6:481-487, 1992

6. Broshek DK, Samples H, Beard J, Goodkin HP: Current practices of the child neurologist in managing sports concussion. $\mathbf{J}$ Child Neurol [epub ahead of print], 2012

7. Cantu RC, Guskiewicz K, Register-Mihalik JK: A retrospective clinical analysis of moderate to severe athletic concussions. PM R 2:1088-1093, 2010

8. Cantu RC, Register-Mihalik JK: Considerations for return-toplay and retirement decisions after concussion. PM R 3 (10 Suppl 2):S440-S444, 2011

9. Capruso DX, Levin HS: Cognitive impairment following closed head injury. Neurol Clin 10:879-893, 1992

10. Chen JK, Johnston KM, Collie A, McCrory P, Ptito A: A validation of the post concussion symptom scale in the assessment of complex concussion using cognitive testing and functional MRI. J Neurol Neurosurg Psychiatry 78:1231-1238, 2007

11. Choe MC, Babikian T, DiFiori J, Hovda DA, Giza CC: A pediatric perspective on concussion pathophysiology. Curr Opin Pediatr 24:689-695, 2012

12. Covassin T, Elbin RJ, Harris W, Parker T, Kontos A: The role of age and sex in symptoms, neurocognitive performance, and postural stability in athletes after concussion. Am J Sports Med 40:1303-1312, 2012

13. Covassin T, Elbin RJ III, Larson E, Kontos AP: Sex and age differences in depression and baseline sport-related concussion neurocognitive performance and symptoms. Clin J Sport Med 22:98-104, 2012

14. Dick R, Ferrara MS, Agel J, Courson R, Marshall SW, Hanley MJ, et al: Descriptive epidemiology of collegiate men's football injuries: National Collegiate Athletic Association Injury Surveillance System, 1988-1989 through 2003-2004. J Athl Train 42:221-233, 2007

15. Englander J, Hall K, Stimpson T, Chaffin S: Mild traumatic brain injury in an insured population: subjective complaints and return to employment. Brain Inj 6:161-166, 1992

16. Field M, Collins MW, Lovell MR, Maroon J: Does age play a role in recovery from sports-related concussion? A comparison of high school and collegiate athletes. J Pediatr 142:546553,2003

17. Fourtassi M, Hajjioui A, Ouahabi AE, Benmassaoud H, HajjajHassouni N, Khamlichi AE: Long term outcome following mild traumatic brain injury in Moroccan patients. Clin Neurol Neurosurg 113:716-720, 2011

18. Gessel LM, Fields SK, Collins CL, Dick RW, Comstock RD: Concussions among United States high school and collegiate athletes. J Athl Train 42:495-503, 2007

19. Guerriero RM, Proctor MR, Mannix R, Meehan WP III: Epi- demiology, trends, assessment and management of sport-related concussion in United States high schools. Curr Opin Pediatr 24:696-701, 2012

20. Gilchrist J, Thomas KE, Xu L, McGuire LC: Nonfatal traumatic brain injuries related to sports and recreation activities among persons aged $\leq 19$ years-United States, 2001-2009. MMWR Morb Mortal Wkly Rep 60:1337-1342, 2011

21. Iverson GL, Lovell MR, Collins MW: Interpreting change on ImPACT following sport concussion. Clin Neuropsychol 17:460-467, 2003

22. Kerr ZY, Marshall SW, Harding HP Jr, Guskiewicz KM: Nine-year risk of depression diagnosis increases with increasing self-reported concussions in retired professional football players. Am J Sports Med 40:2206-2212, 2012

23. Kirkwood MW, Yeates KO, Wilson PE: Pediatric sport-related concussion: a review of the clinical management of an oftneglected population. Pediatrics 117:1359-1371, 2006

24. Lovell MR, Iverson GL, Collins MW, Podell K, Johnston KM, Pardini D, et al: Measurement of symptoms following sportsrelated concussion: reliability and normative data for the postconcussion scale. Appl Neuropsychol 13:166-174, 2006

25. Marar M, Mcllvain NM, Fields SK, Comstock RD: Epidemiology of concussions among United States high school athletes in 20 sports. Am J Sports Med 40:747-755, 2012

26. Maroon JC, Lovell MR, Norwig J, Podell K, Powell JW, Hartl R: Cerebral concussion in athletes: evaluation and neuropsychological testing. Neurosurgery 47:659-672, 2000

27. McCrory P, Meeuwisse W, Johnston K, Dvorak J, Aubry M, Molloy M, et al: Consensus statement on concussion in sportthe 3rd International Conference on Concussion in Sport held in Zurich, November 2008. PM R 1:406-420, 2009

28. Powell JW, Barber-Foss KD: Injury patterns in selected high school sports: a review of the 1995-1997 seasons. J Athl Train 34:277-284, 1999

29. Rutherford WH: Sequelae of concussion caused by minor head injuries. Lancet 309:1-4, 1977

30. Schatz P, Moser RS, Covassin T, Karpf R: Early indicators of enduring symptoms in high school athletes with multiple previous concussions. Neurosurgery 68:1562-1567, 2011

31. Schatz P, Pardini JE, Lovell MR, Collins MW, Podell K: Sensitivity and specificity of the ImPACT Test Battery for concussion in athletes. Arch Clin Neuropsychol 21:91-99, 2006

32. Sim A, Terryberry-Spohr L, Wilson KR: Prolonged recovery of memory functioning after mild traumatic brain injury in adolescent athletes. J Neurosurg 108:511-516, 2008

33. Sosin DM, Sniezek JE, Thurman DJ: Incidence of mild and moderate brain injury in the United States, 1991. Brain Inj 10:47-54, 1996

34. Zuckerman SL, Lee YM, Odom MJ, Solomon GS, Forbes JA, Sills AK: Recovery from sports-related concussion: days to return to neurocognitive baseline in adolescents versus young adults. Surg Neurol Int 3:130, 2012

Manuscript submitted November 29, 2012.

Accepted July 8, 2013.

Please include this information when citing this paper: published online September 24, 2013; DOI: 10.3171/2013.7.PEDS12572.

Address correspondence to: Scott L. Zuckerman, M.D., 1500 21st Ave. South, Ste. 4340, Nashville, TN 37212. email: scott.zuckerman @vanderbilt.edu. 